\title{
Survival from malignant mesothelioma: where are we now?
}

\author{
${ }^{1} \mathrm{AD}$ Marshall, ${ }^{2} \mathrm{HK}$ Bayes, ${ }^{3} \mathrm{~J}$ Bardgett, ${ }^{4} \mathrm{~S}$ Wedderburn, ${ }^{5} \mathrm{KM}$ Kerr, ${ }^{6} \mathrm{GP}$ Currie \\ ${ }^{1}$ StR Respiratory Medicine, ${ }^{3}$ ACCS trainee, ${ }^{4}$ Foundation trainee, ${ }^{6}$ Consultant Respiratory Physician, Chest Clinic C, Aberdeen Royal Infirmary, \\ Aberdeen, UK; ${ }^{5}$ Consultant Pathologist, Pathology Department, Aberdeen Royal Infirmary, Aberdeen, UK; ${ }^{2}$ Clinical Lecturer in Respiratory \\ Medicine, Institute of Infection, Inflammation and Immunity, University of Glasgow, Glasgow, UK
}

\begin{abstract}
Background: The prognosis of malignant pleural mesothelioma has traditionally been poor.Whether this remains the case compared to historical data and within a specific geographical location is uncertain. Knowledge of predictive factors for survival with malignant pleural mesothelioma is also inadequate.

Methods: We conducted a retrospective local database analysis to determine overall prognosis of patients with malignant pleural mesothelioma and evaluate the influence of demographic characteristics, histological subtype and laboratory parameters. Patients with histological diagnoses of malignant pleural mesothelioma held on the NHS Grampian pathology database between 2002 and 2012 were analysed. Data on baseline demographics, mode of diagnosis, histological sub-type, and survival and serum laboratory parameters, were analysed; time to death was examined using Cox regression analyses.

Results: A total of II4 patients with malignant pleural mesothelioma were included in the analysis. The median survival was 345 days (IQR 99-600). Sarcomatoid malignant pleural mesothelioma carried a significantly worse prognosis with median survival of 125 days (IQR 44-289) vs 334 days (IQR I26-7I5) for biphasic, 4 I 2 days (IQR 20 I-656) for epithelioid and 345 days (IQR 99-600) for those with no definitive typing. Individuals who did not receive chemotherapy experienced a significantly worse prognosis (hazard ratio 2.7; $95 \% \mathrm{Cl}$ I.5-4.7; $\mathrm{p}=0.00 \mathrm{I}$ ), while a low albumin and raised urea at time of diagnosis were also associated with a significantly poorer prognosis.

Conclusion: The survival of patients with malignant pleural mesothelioma remains poor and typically around I year. The presence of raised urea and low albumin is associated with a poorer prognosis, while patients with a good performance status and few co-morbidities should be encouraged to receive chemotherapy.
\end{abstract}

KEYWORDS mesothelioma, prognosis, prognostic factors

DECLARATION OF INTERESTS No conflict of interest declared.

\author{
Correspondence to GP Currie \\ Chest Clinic C \\ Aberdeen Royal Infirmary \\ Foresterhill \\ Aberdeen AB25 2ZN \\ UK
}

e-mail graeme.currie@nhs.net

\section{INTRODUCTION}

Malignant pleural mesothelioma (MPM) is an aggressive cancer that occurs usually as a consequence of inhalation of asbestos fibres.' It typically affects males following a latent period after occupational exposure, although para-occupational (especially in females) and environmental exposure causing asbestos-related disorders are well recognised. ${ }^{2}$ The incidence of MPM has increased over the past few decades and it has been suggested that annual deaths will peak in the UK between $20 \mathrm{II}$ and $2015{ }^{3}$ A paucity of older studies prior to widespread introduction of more rapid diagnostic tools and advances in chemotherapy regimes - have reported survival data, ${ }^{4-6}$ with median prognosis considered to be 8-14 months following diagnosis. ${ }^{4,-8}$
Specific clinical and laboratory prognostic factors have not been exhaustively evaluated in recent years, although the sarcomatoid subtype generally heralds a poor prognosis. ${ }^{8}$ Prognosis and prognostic factors in one locality - especially with different degrees and types of asbestos exposure - may not necessarily be assumed to be the same in others and uncertainty must therefore exist when attempts are made to anticipate survival from patient to patient and from one geographical area to the next. Prognosis of MPM is also vital to consider in patients prior to consideration of systemic treatment; regimes associated with unacceptable morbidity and mortality and inherent risk of hospital admission should ideally be avoided in individuals unlikely to live long following diagnosis. 
The primary aim of this study was to evaluate the overall prognosis of patients from the time of diagnosis with biopsy-proven MPM within our own institution. As a secondary aim, we wished to explore the influence of individual patient-specific characteristics in an attempt to help guide patients and their families regarding likely life expectancy following diagnosis.

\section{METHODS}

A retrospective analysis of the NHS Grampian pathology database was performed to identify all patients histologically diagnosed ante-mortem with MPM between January 2002 and December 2012 (inclusive); all patients (at the time of diagnostic procedure) were resident within the catchment area of NHS Grampian in the north east of Scotland, which covers a population of approximately 500,000. Demographic data, mode of diagnosis, histological subtype, survival and serum laboratory parameters at the time of diagnosis were then extracted from the database. Survival time was calculated from date of diagnosis until death from any cause or until last follow-up in censored patients, with follow-up of patients remaining alive performed until March 2015.

A Kaplan-Meier with Mantel-Cox log-rank analysis was performed on MPM survival data. A Cox proportional hazards model with backwards stepwise selection was used to assess the effect of variables on survival from MPM. The parameters of age, gender, pathological type, receipt of chemotherapy, white cell count, neutrophil leukocyte ratio (NLR; obtained by dividing the blood neutrophil count by the blood lymphocyte count), serum albumin, urea, creatinine and C-reactive protein were included in the multivariate analysis. A C-reactive protein less than the limits of detection were assigned a value of zero. P-values $>0.05$ were deemed non-significant. Statistical analysis was performed using Prism version 6 and SPSS version 21 .

\section{RESULTS}

\section{Patient demographics}

A total of 130 patients were identified as having MPM within the study period. Post-mortem diagnosis was made in six patients and no accurate follow-up data were available in ten, meaning that II4 patients with MPM diagnosed on histological specimens ante-mortem were included in the survival analysis.

Patients were predominantly male $(n=96,84 \%)$ with a right-sided ( $n=66,58 \%$ ) MPM, and the median age was 70 years at diagnosis (IQR 63-76). The most common pathological subtype was epithelioid ( $n=64,56 \%$ ), followed by biphasic $(n=18,16 \%)$ and sarcomatoid $(n=$ $18,16 \%)$, while $12 \%(n=14)$ had no definitive typing.

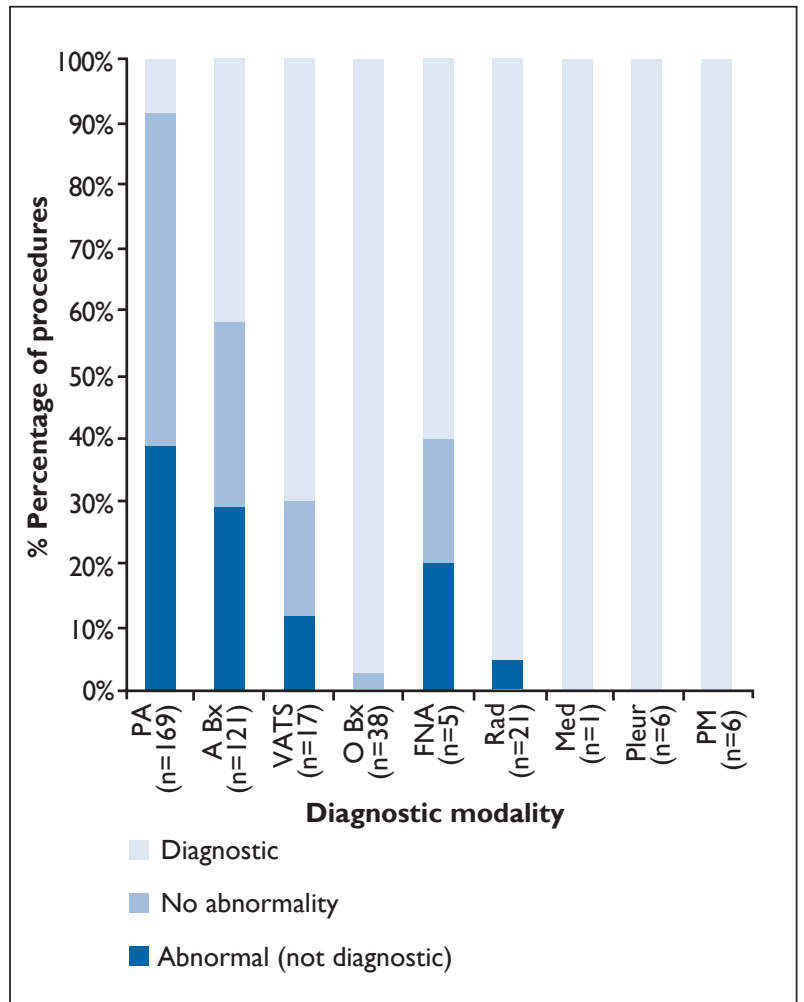

FIGURE I Bar graph showing the numbers of each diagnostic procedure carried out and their comparative diagnostic yield. PA: pleural aspirate; $A B x$ : Abrams biopsy; VATS: video assisted thoracoscopy; OBx: open biopsy; FNA: fine needle aspirate; Rad: radiologically guided biopsy, Med: mediastinoscopy; Pleur: pleurectomy; PM: postmortem examination

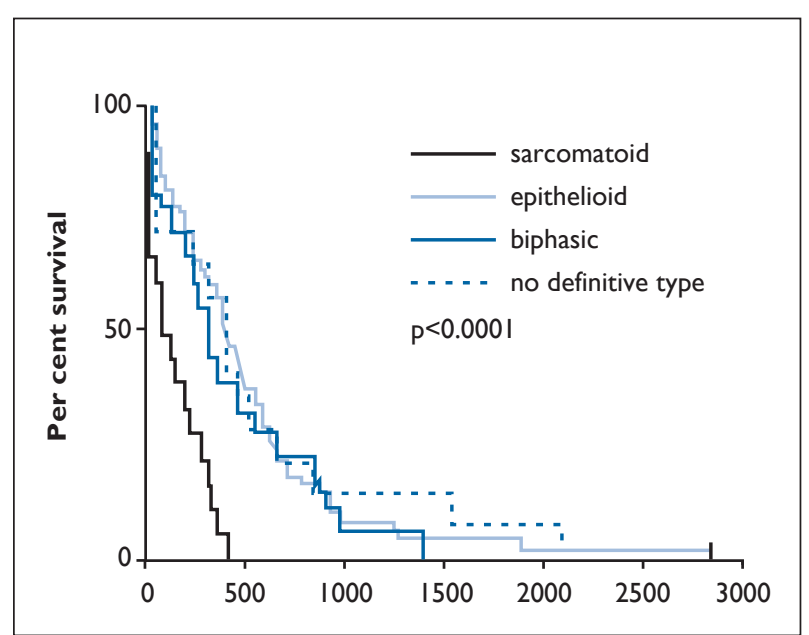

FIGURE 2 Survival in patients with sarcomatoid MPM versus all other groups; $\mathrm{p}$-value relates to Kaplan-Meier analysis with log-rank test

Pathological diagnosis was obtained via a variety of techniques (Figure I). The most common diagnostic modalities were pleural aspiration and Abrams biopsy; however these demonstrated low positive diagnostic yield ( $9 \%$ and $41 \%$, respectively). Targeted biopsy techniques demonstrated the highest positive yield rates. 
TABLE I COX proportional hazards model for malignant pleural mesothelioma

\begin{tabular}{|c|c|c|}
\hline Variable & $\begin{array}{l}\text { Hazard Ratio } \\
(95 \% \mathrm{Cl})\end{array}$ & p-value \\
\hline $\begin{array}{l}\text { Gender } \\
\text { male vs female }\end{array}$ & $\begin{array}{c}2.2 \\
(1.0-4.7)\end{array}$ & 0.05 \\
\hline \begin{tabular}{|l|} 
Pathological subtype \\
(versus epithelioid)
\end{tabular} & & \\
\hline Sarcomatoid & $6.6(3.2-13.5)$ & 0.0001 \\
\hline Biphasic & $2.2(1.2-4.3)$ & 0.016 \\
\hline No definitive type & $1.4(0.6-3.2)$ & 0.47 \\
\hline $\begin{array}{l}\text { Chemotherapy } \\
\text { no chemotherapy vs } \\
\text { chemotherapy given }\end{array}$ & $2.7(1.5-4.7)$ & 0.001 \\
\hline $\begin{array}{l}\text { Serum urea } \\
\text { (per mmol/L) }\end{array}$ & I.I (I.0-I.2) & 0.038 \\
\hline $\begin{array}{l}\text { Serum albumin } \\
\text { (per g/L) }\end{array}$ & $0.90(0.85-0.95)$ & 0.0001 \\
\hline
\end{tabular}

Overall median survival of all patients with MPM was 345 days (IQR 99-600) from diagnosis. Sarcomatoid MPM carried a significantly worse prognosis with median survival of I25 days (IQR 44-289), compared to 334 days (IQR I26-7I5) for biphasic, 4I2 days (IQR 20I656) for epithelioid and 345 days (IQR 99-600) for those with no definitive typing (Figure 2; $\mathrm{p}<0.000 \mathrm{I}$ ).

\section{Parameters influencing MPM survival}

A multivariate Cox proportional hazards model identified the parameters independently associated with survival, with covariates not significantly influencing outcome removed by back selection. Each histological subtype was compared with epithelioid MPM as this independently carried the best survival. The final model is shown in Table I. Age, white cell count, C-reactive protein, creatinine and NLR were not significantly associated with MPM survival, while male gender, sarcomatoid subtype, raised serum urea and lower serum albumin levels were independently associated with poorer survival in MPM. Sarcomatoid subtype was the most hazardous variable in the model with a hazard ratio of $6.6(95 \% \mathrm{Cl} 3.2-13.6)$. Although identified as independent predictors, raised urea and reduced albumin were only weakly associated with a poorer prognosis with hazard ratios of $I . I$ and 0.9 , respectively.

Whether or not chemotherapy had been received could be ascertained in 85 individuals. Using Kaplan-Meier analysis (Figure 3), those receiving chemotherapy experienced a significantly superior prognosis; median survival in those receiving chemotherapy $(n=47)$ was 425 days (IQR 3I4-704) vs I24 days (IQR 6I-347) in others $(n=38)(p=0.0006)$. The positive independent effect of chemotherapy on MPM survival was further verified in the Cox model (Table I) with a hazard ratio of 2.7 when chemotherapy was not given $(p=0.00 \mathrm{I})$.

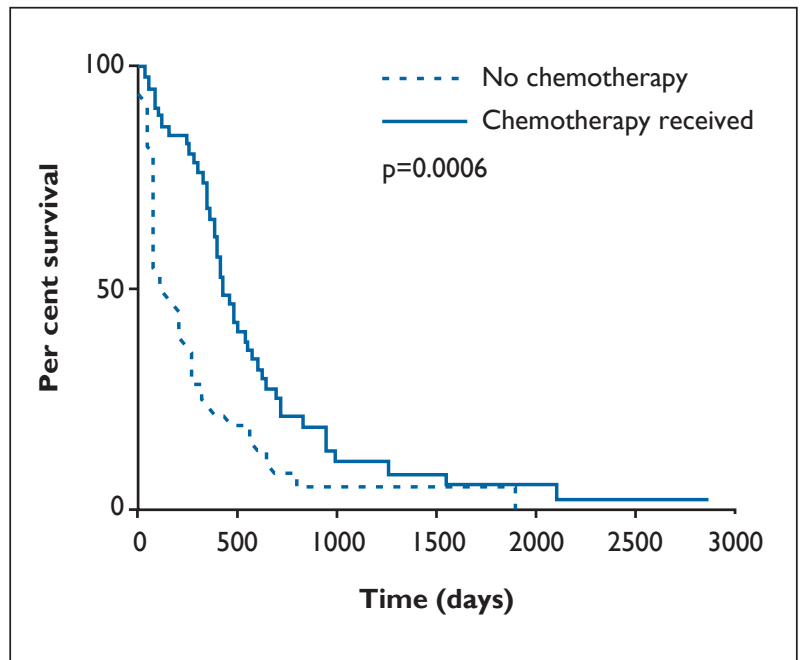

FIGURE 3 Survival in patients with MPM receiving chemotherapy; p-value relates to Kaplan-Meier analysis with log-rank test

\section{CONCLUSION}

We have shown that across an II-year period, overall median survival in patients with MPM was approximately I year. It is disappointing that, following publication of previous data relating to patients well over a decade ago, the long-term prognosis of MPM remains poor.' The reasons behind this are likely to be multifactorial. For example, since MPM typically presents in the seventh decade, many patients will have other significant co-morbidities and respiratory failure. Typically these will be from concomitant chronic obstructive pulmonary disease, meaning that more aggressive treatment regimes may be precluded, while MPM itself generally presents as a fairly advanced and incurable disease.

Moreover, in our own cohort from 2006 onwards, only around $40 \%$ of patients were considered sufficiently fit for or agreed to treatment with chemotherapy. Unlike early stage lung cancer in patients with good performance status (PS), radical surgery in MPM is generally not undertaken. In a multi-centre trial, 50 patients were randomised to undergo extra-pleural pneumonectomy (EPP) or not. ${ }^{10}$ Those having surgery experienced a poorer median survival of 14 months while the corresponding value was 20 months in the 'no EPP' group. As many as ten patients in the former group experienced a serious adverse effect vs two in the latter.

Further, in a recent retrospective study evaluating outcomes of 1365 patients, EPP and extended pleurectomy decortication conferred no survival benefit vs medical therapy alone." However, there are some encouraging data suggesting that, with accurate preoperative tissue diagnosis, nodal staging and induction chemotherapy prior to radical surgery, it is possible to achieve long term survival. ${ }^{12}$ 
AD Marshall, HK Bayes, J Bardgett et al.

As with other studies, ${ }^{6,8}$ we demonstrated that the sarcomatoid subtype was an adverse prognostic factor, while we also identified male gender, low serum albumin and raised serum urea as predictors of a poorer survival. Although in isolation the ability of these prognostic factors to predict prognosis is not possible, their presence may provide the clinician with additional information when discussing life-expectancy with patients and their families. Moreover, low albumin and elevated urea may represent surrogate markers of poor nutritional status and dehydration respectively, and perhaps raise the notion that these 'correctable' factors are explored.

The retrospective nature of our data means that other potentially useful prognostic factors such as smoking history and PS at time of diagnosis were not available. A further drawback is that patients were retrospectively identified from a pathology database; although we feel we captured all those with histologically-proven MPM. It is likely that some patients - especially those with a poor PS, co-morbidities and advanced age - over the II-year period studied did have mesothelioma but a tissue diagnosis was not pursued meaning that data capture and analysis were incomplete. However, it could be argued that with identification of these patients, the prognosis would have been even poorer than that calculated.

\section{REFERENCES}

I Currie GP, Watt SJ, Maskell NA. An overview of how asbestos exposure affects the lung. BMJ 2009; 339: b3209. http://dx.doi. org/I0.1 I36/bmj.b3209

2 Howel D, Arblaster L, Swinburne L et al. Routes of asbestos exposure and the development of mesothelioma in an English region. Occup Environ Med 1997; 54: 403-9.

3 Hodgson JT, McElvenny DM, Darnton AJ et al. The expected burden of mesothelioma mortality in Great Britain from 2002 to 2050. Br J Cancer 2005; 92: 587-93.

4 British Thoracic Society Standards of Care Committee. BTS statement on malignant mesothelioma in the UK, 2007. Thorax 2007; 62 Suppl 2: ii I-iil9.

5 Ribak J, Selikoff IJ. Survival of asbestos insulation workers with mesothelioma. Br J Ind Med 1992; 49: 732-5.

6 Yates DH, Corrin B, Stidolph PN et al. Malignant mesothelioma in south east England: clinicopathological experience of 272 cases. Thorax 1997; 52: 507-12.

7 Hulks G, Thomas JS, Waclawski E. Malignant pleural mesothelioma in western Glasgow 1980-6. Thorax 1989; 44: 496-500.

8 Van Gelder T, Damhuis RA, Hoogsteden HC. Prognostic factors and survival in malignant pleural mesothelioma. Eur Respir J 1994; 7: 1035-8.

9 Beckett P, Woolhouse I, Stanley R et al. PI An analysis of 8503 cases of mesothelioma from the national lung cancer audit. Thorax 20।3; 68:A75.
Recently, based on data derived from three large international cohorts of patients with a malignant pleural effusion of any cause, the LENT prognostic score (pleural fluid lactate dehydrogenase, Eastern Cooperative Oncology Group PS, NLR and tumour type) was developed. ${ }^{13}$ This demonstrated that only $65 \%$ of patients with a high LENT score survived I month from diagnosis and only $3 \%$ survived 6 months. Moreover, the LENT score was considered to be significantly superior at predicting survival compared with Eastern Cooperative Oncology Group PS at I, 3 and 6 months. Pleural fluid lactate dehydrogenase and PS were unfortunately not available in the majority of our cohort, although we did demonstrate that the peripheral blood NLR in isolation was not associated with differences in prognosis in patients with MPM. Identification of further biological prognostic factors in MPM is thus warranted, with early studies of biomarkers currently being explored. ${ }^{14}$

In summary, our data reinforce the fact that prognosis of MPM in general remains poor and unchanged over the years. Moreover, we highlight that factors associated with a poorer prognosis include the sarcomatoid subtype, male gender, elevated urea and low albumen, while patients who receive chemotherapy tend to experience a more favourable prognosis.
10 Treasure T, Lang-Lazdunski L, Waller D et al. Extra-pleural pneumonectomy versus no extra-pleural pneumonectomy for patients with malignant pleural mesothelioma: clinical outcomes of the Mesothelioma and Radical Surgery (MARS) randomised feasibility study. Lancet Oncol 20II; 12: 763-72. http://dx.doi. org/I0.10I6/SI470-2045(II)70I49-8

I I Bovolato P, Casadio C, Bille A et al. Does surgery improve survival of patients with malignant pleural mesothelioma?: a multicenter retrospective analysis of 1365 consecutive patients. J Thorac Oncol 2014; 9: 390-6. http://dx.doi.org/10.1097/JTO.0000000000000064

12 Nakas A, Waller D. Predictors of long-term survival following radical surgery for malignant pleural mesothelioma. Eur J Cardiothorac Surg 20I4; 46: 380-5. http://dx.doi.org/I0.I093/ejcts/ ezt664

13 Clive AO, Kahan BC, Hooper CE et al. Predicting survival in malignant pleural effusion: development and validation of the LENT prognostic score. Thorax 2014; 69: 1098-104. http://dx.doi. org/I0.I I36/thoraxjnl-20I4-205285

14 Matsumoto S, Nabeshima K, Hamasaki M et al. Upregulation of microRNA-3I associates with a poor prognosis of malignant pleural mesothelioma with sarcomatoid component. Med Oncol 20|4; 31: 303. http://dx.doi.org/I0.1007/s | 2032-0|4-0303-2 